\title{
GESTÃO DO CONHECIMENTO COMO APOIO À MELHORIA CONTÍNUA: UM ESTUDO DE CASO EM UMA EMPRESA DE TELECOMUNICAÇÕES
}

\author{
GESTIÓN DEL CONOCIMIENTO COMO APOYO A LA \\ MEJORA CONTINUA: UN ESTUDIO DE CASO EN UNA \\ EMPRESA DE TELECOMUNICACIONES ${ }^{1}$
}

Andréia de Abreu-andreiabreu11@gmail.com Doutoranda em Engenharia de Produção (UFSCar). Professora do

Curso de Tecnologia em Gestão Empresarial da Faculdade de Tecnologia de Garça (FATEC).

\begin{abstract}
Cássia Regina Bassan de Moraes-crbassan@gmail.com Doutora em Ciência da Informação (UNESP/ Marília). Coordenadora e professora do Curso de Tecnologia em Gestão Empresarial da Faculdade de Tecnologia de Garça (FATEC)

Luana Maia Woida-luanamwoida@yahoo.com.br Doutora em Ciência da Informação (UNESP/ Marília). Professora do Curso de Tecnologia em Gestão Empresarial da Faculdade de Tecnologia de Garça (FATEC)
\end{abstract}

\section{RESUMO}

Introdução: A gestão da melhoria contínua tem se tornado comum, porém as organizações frequentemente encontram dificuldades na implementação do conceito.

\footnotetext{
${ }^{1}$ Resultados parciais obtidos da pesquisa "Construção de conhecimento a partir da socialização organizacional: aporte para a inovação na indústria de eletro-eletrônicos de Garça/SP", coordenada pela Dra. Cássia Regina Bassan de Moraes e financiada pelo Conselho Nacional de Desenvolvimento Científico e Tecnológico (CNPq), no de processo: 406214/2012-3, Chamada $\mathrm{MCTI} / \mathrm{CNPq} / \mathrm{MEC} / \mathrm{CAPES} \mathrm{N} \cong 18 / 2012$.
} 
Objetivo: O objetivo desse trabalho é analisar como a gestão do conhecimento contribuiu para o envolvimento das pessoas durante um processo de mudança organizacional voltado à melhoria contínua numa empresa do ramo de telecomunicações.

Metodologia: A pesquisa caracteriza-se dentro de uma abordagem qualitativa, fazendo uso do estudo de caso em uma empresa do ramo de serviços em telecomunicações localizada no interior do estado de São Paulo. Para a coleta de dados foi utilizado roteiro de entrevista, aplicado pessoalmente aos funcionários de nível operacional e gerencial envolvidos nos projetos.

Resultados: A análise dos resultados apontou que o envolvimento das pessoas nas mudanças ocasionadas pelo projeto de melhoria, via valorização do seu conhecimento e estímulo ao aprendizado durante o processo, tiveram influência significativa para o sucesso da implementação e, também, na minimização das resistências oriundas.

Conclusão: Concluiu-se que a gestão do conhecimento proporciona maior envolvimento dos indivíduos nos processos de construção de conhecimento, incentiva comprometimento com ações de externalização, cujos comportamentos provêm de uma base sociocultural favorável à adaptação e à mudança.

Palavras-chave: Gestão do conhecimento. Melhoria contínua. Comprometimento. Mudança organizacional.

\section{INTRODUÇÃO}

Cada vez mais as organizações buscam maior agilidade e rapidez em seus processos para se tornarem competitivas nesse mercado em constantes mutações e transformações. Por constituir-se num conceito simples, de fácil entendimento e de baixo nível de investimento (BESSANT et al., 1994), a busca pela melhoria contínua tem se tornado comum nas organizações como meio para o aumento da competitividade e sobrevivência sustentável (ZAMPINI; TOLEDO, 2010).

Apesar da importância reconhecida, as organizações têm encontrado dificuldades para implementarem o conceito de melhoria contínua: a maior parte das iniciativas são falhas (ANAND et al., 2009; PAY, 2008; ZAMPINI; TOLEDO, 2010). Entre as razões comumente apontadas, destacam-se a falta do entendimento, 0 baixo envolvimento das pessoas nos programas e a ausência de cultura organizacional voltada à melhoria contínua. Portanto, há falta de preparo, no sentido técnico e comportamental, para a mudança inerente a qualquer iniciativa de melhoria (BESSANT et al., 2001).

A capacidade para o alcance dos objetivos das organizações atuais depende de fatores como: conhecimento, capacidade inovativa e pessoas preparadas para atuar no processo de construção e uso de conhecimento para a melhoria contínua. Pode-se com isso afirmar ser o conhecimento um recurso competitivo essencial a 
ser aplicado nas práticas de melhoria contínua (FLEURY; OLIVEIRA, 2001; NONAKA; TAKEUCHI, 1997).

Considerando o envolvimento das pessoas como fundamental para a melhoria contínua (BESSANT et al., 2001; CAFFIN; BESSANT, 1996; SHIBA; GRAHAM; WALDEN, 1993), vários autores destacam o papel da aprendizagem organizacional como fundamental, e também dinamizador, desse envolvimento (ANAND et al., 2009; BESSANT; FRANCIS, 1999; TRANFIELD et al., 2000), afirmando que a melhoria contínua é somente alcançada quando o aprendizado ocorre de modo contínuo e sistematizado na organização.

Por ser inerente à melhoria contínua, a mudança sempre provoca o desequilíbrio na organização e, consequentemente, no cotidiano das pessoas que trabalham nela. Portanto, para que haja a mudança organizacional é preciso que as pessoas ligadas à empresa entendam que tais processos de mudanças realmente são necessários para a organização. Outro ponto a ser analisado é a prontidão, ou seja, é necessário comprometimento das pessoas envolvidas com tais processos.

A predisposição para a mudança por parte da organização tem forte dependência das características e dos paradigmas de sua cultura organizacional. Mudanças ocorridas em função da melhoria contínua necessitam de uma série de ações e recursos de apoio. Nisso, a relação da cultura organizacional com a melhoria contínua se dá em dois aspectos: primeiro, na existência de uma cultura organizacional voltada à busca constante por melhoria a partir da consciência da necessidade de mudança em função das influências do mercado e da busca pela competitividade; segundo, na transferência desse estado de consciência para ações concretas, de ordem estrutural, técnica e comportamental (JAGER et al., 2004).

Nesse contexto, o objetivo desse artigo foi analisar como a gestão do conhecimento contribuiu para o envolvimento das pessoas durante um processo de mudança organizacional voltado à melhoria contínua numa empresa do ramo de telecomunicações.

Para tanto, o trabalho estrutura-se em duas etapas: bibliográfica e descritiva. A bibliográfica é constituída de revisão de literatura sobre os temas gestão do conhecimento, aprendizagem organizacional, mudança e cultura organizacional e a ligação deles com a melhoria contínua. A descritiva consta de uma pesquisa de 
Andréia de Abreu; Cássia Regina Bassan de Moraes; Luana Maia Woida

Gestão do conhecimento como apoio à melhoria contínua: um estudo de caso em uma empresa de telecomunicações

campo, na forma de um estudo de caso, realizada numa empresa de telecomunicações localizada no interior do estado de São Paulo.

A próxima seção trata da revisão de literatura, seguida da apresentação do método de pesquisa utilizado para o estudo de caso. Posteriormente, há a apresentação e discussão dos resultados encontrados. A última seção apresenta as considerações finais.

\section{REVISÃO DE LITERATURA}

\subsection{O Conhecimento nas Organizações}

Nas últimas décadas, a Gestão do Conhecimento tem se tornado um dos temas mais importantes nos estudos e discussões sobre a gestão empresarial. Em muitas empresas criaram-se novos cargos como "Gerente de Gestão de Conhecimento" ou funções implícitas em cargos de qualidade, tecnologia da informação, educação e treinamento, entre outras, utilizando-se de ferramentas da Gestão do Conhecimento para controlar, sintetizar e gerenciar as informações e conhecimentos da empresa.

Sua aplicação tem sido percebida em diferentes iniciativas de inovação, como por exemplo, nos processos de desenvolvimento de produto (SILVA; ROZENFELD, 2007). Com isso, é possível que a empresa utilize o aprendizado adquirido em trabalhos e eventos passados para diminuir o tempo e aperfeiçoar processos e projetos em execução e em planejamento (TERRA, 2001; TERRA; GORDON, 2011).

Logo, surge a compreensão que a Gestão do Conhecimento implica na coordenação sistêmica de esforços nos planos organizacionais e individuais, estratégicos e operacionais e nas normas formais e informais entre as várias dimensões da empresa (TERRA, 2001).

Valentim (2008) define conhecimento como sendo o produto de um sujeito cognitivo que a partir da internalização de diferentes informações e percepções elabora ou reelabora o seu novo conhecimento. A autora afirma que em ambientes organizacionais o conhecimento construído por um indivíduo alimenta a construção do conhecimento coletivo e, por outro lado, o conhecimento coletivo alimenta a construção do conhecimento individual. 
$\mathrm{Na}$ mesma linha de raciocínio, conhecimento como reflexão sobre a experiência, Davenport e Prusak (1998) apresentam a definição de conhecimento como sendo uma mistura fluida de experiência condensada, valores, informação contextual e insight experimentado, a qual proporciona uma estrutura para a avaliação e incorporação de novas experiências e informações. Nas organizações, o conhecimento costuma estar embutido não só em documentos ou repositórios, mas também em rotinas, processos, práticas e normas organizacionais.

Pode-se perceber que tanto Valentim (2008) como Davenport e Prusak (1998) convergem para compreender o conhecimento como resultado de um processo interno do indivíduo, que processa a realidade a partir de suas próprias experiências e transmite o resultado aos que com ele convivem, num espiral que tende ao infinito.

Para Takeuchi e Nonaka (2008), há dois tipos de conhecimento: o explícito e o tácito. O conhecimento explícito é aquele que pode ser expresso em palavras, números ou sons, e compartilhado na forma de dados, fórmulas científicas, recursos visuais, especificações de produtos ou manuais. Portanto, pode ser rapidamente transmitido aos indivíduos, de modo formal e sistematicamente. O conhecimento tácito, por outro lado, não é facilmente visível e explicável. Pelo contrário, é altamente pessoal e difícil de formalizar, tornando-se sua comunicação e compartilhamento dificultoso. As intuições e os palpites subjetivos estão sob a rubrica do conhecimento tácito.

Nonaka e Takeuchi (2008) estruturam duas dimensões do conhecimento:

a) dimensão ontológica - uma organização não pode criar conhecimento sem indivíduos, pois o conhecimento só é criado por indivíduos. A criação do conhecimento organizacional deve ser entendida como um processo que amplia organizacionalmente o conhecimento criado pelos indivíduos, cristalizando-o como parte da rede de conhecimentos da organização.

b) dimensão epistemológica - baseada na distinção estabelecida por Polanyi (1966 apud NONAKA; TAKEUCHI, 2008) entre conhecimento tácito e conhecimento explícito. O conhecimento explícito é transmissível em linguagem formal e sistemática e pode ser facilmente transmitido entre os indivíduos. O conhecimento tácito é pessoal, específico ao contexto, difícil de ser formulado e transmitido, pois envolve fatores intangíveis. 
Para outros autores acima citados, esses dois tipos de conhecimento se complementam e a interação entre ambos gera a dinâmica da criação. Sendo assim, a criação do conhecimento ocorre em três níveis: indivíduo, grupo e organização. As duas formas de interação - entre o conhecimento tácito e o conhecimento explícito e entre o indivíduo e a organização - levarão aos quatro processos principais da conversão do conhecimento que, juntos, constituem a criação do conhecimento: 1. Socialização: do tácito para o tácito; 2. Externalização: do tácito para o explícito; 3. Combinação: do explícito para o explícito; 4. Internalização: do explícito para o tácito.

Pérez-Montoro Gutiérrez (2008) propõe a distinção de seis tipos diferentes de conhecimento, cada um deles dotados de uma natureza especial, e que podem ser agrupados em três pares: (a) conhecimento tácito/conhecimento explícito; (b) conhecimento individual/conhecimento organizacional ou corporativo e (c) conhecimento interno/conhecimento externo.

Ao explicar as relações entre 0 primeiro par (conhecimento tácito/conhecimento explícito), Pérez-Montoro Gutiérrez (2008) utiliza a conceituação estabelecida por Nonaka e Takeuchi (2008). Ao explicitar as diferenças entre conhecimento individual e conhecimento organizacional ou corporativo, PérezMontoro Gutiérrez (2008) apresenta as seguintes definições:

- conhecimento individual: todo aquele conhecimentos que possui, em sua mente, um membro de uma organização, sendo formado pela síntese de todos os conhecimentos tácitos e explícitos que possui. Suas habilidades individuais, seus contatos e relações pessoais ou seus conhecimentos técnicos podem ser identificados como parte desse conhecimento individual.

- conhecimento organizacional: aquele atribuído e possuído por uma organização, comumente representado na forma de documentos. As bases de dados adquiridas por uma organização, ou a propriedade intelectual e as patentes que esta desenvolve são dois claros exemplos deste tipo de conhecimento. 
O autor acrescenta que, em um sentido estrito, o conhecimento organizacional ou corporativo não existe de fato. Se o conhecimento é um tipo especial de estado mental que possui uma pessoa, as organizações, por não poder ter estados mentais (por não ter um cérebro material que possa mantê-los, literalmente), não podem tampouco possuir nenhum tipo de conhecimento. De qualquer forma, em um sentido lato, fala-se de conhecimento organizacional ou corporativo para referir-se àquelas informações que se correspondem com algum conhecimento concreto (que alguém possui), que se encontra representado e de cuja representação a organização é proprietária.

A essa ideia, Davenport e Prusak (1998) acrescentam que uma organização, por ser concebida como um conjunto de pessoas organizadas para produzir algo, tem sua capacidade produtiva altamente dependente daquilo que ela sabe e do conhecimento subjacentes nas rotinas e equipamentos de produção. Consequentemente, o ativo material de uma empresa só terá valor real se as pessoas souberem o que fazer com ele. Se "saber fazer" define o que a empresa é, então o conhecimento realmente é a empresa num sentido importante.

Quanto ao último par dos tipos de conhecimento, Pérez-Montoro Gutiérrez (2008) define o conhecimento interno como aquele fundamental para o funcionamento e alcance dos objetivos da organização, sendo com isso bastante crítico e importante. Já o conhecimento externo é aquele que uma organização se utiliza para relacionar-se com outras organizações.

A atribuição de significado ou reconhecimento de que a informação precisa ter sentido é uma ação ou processo realizado pelas pessoas e o princípio para se considerar uma organização capaz de trabalhar o conhecimento. Choo (2003) aborda os diversos aspectos que se relacionam à organização do conhecimento, com o propósito de esclarecer as possibilidades e os caminhos que as organizações possuem para executarem a criação e o uso do conhecimento de maneira estratégica para a ação.

Choo (2003) segue um modelo que enfatiza a ação e a prática, isto é, o uso e a criação do conhecimento a partir de ações realizadas sobre informações advindas do ambiente e absorvidas pelos indivíduos. Reconhece a criação de conhecimento como uma ocorrência individual, mas volta-se para a cultura da organização para 
contextualizar o sentido atribuído à informação e a utilidade prevista para o conhecimento produzido.

A criação e o uso estratégico do conhecimento dependem de processos cognitivos (CHOO, 2003). Choo adota e apoia suas explicações a respeito da abordagem estratégica da criação e do uso da informação, basicamente em três esferas ou arenas. A primeira relaciona-se ao uso da informação para atribuir sentido às mudanças do ambiente e constitui um recurso indispensável para compreender os movimentos, demandas e exigências associadas a cada stakeholder. Portanto, o objetivo transcende os prognósticos sobre tendências e mudanças, e usa as informações para criar um arcabouço de conhecimentos relevantes, que possibilitem adaptação e à dinâmica do ambiente. Nesse caso, destacando-se como fundamental para a melhoria contínua, porque permite usar as informações para perceber e atuar junto às mudanças do ambiente.

A segunda arena ou dimensão de criação e uso da informação é atribuída à maneira como criar, organizar e processar as informações, com o intuito de produzir conhecimentos fazendo uso do processo de aprendizado (CHOO, 2003). Esfera que sintetiza a função da gestão do conhecimento, mostrando-a como importante para gerar aprendizado.

A busca e a avaliação das informações também são consideradas etapas fundamentais e, por isso, constituem a terceira arena ou dimensão para o uso estratégico da informação. A fim de resumir as ideias desse autor, essenciais para compreender a dinâmica da informação e do conhecimento no ambiente organizacional, apresenta-se a Figura 1.

conhecimento

Figura 1 - Organização do

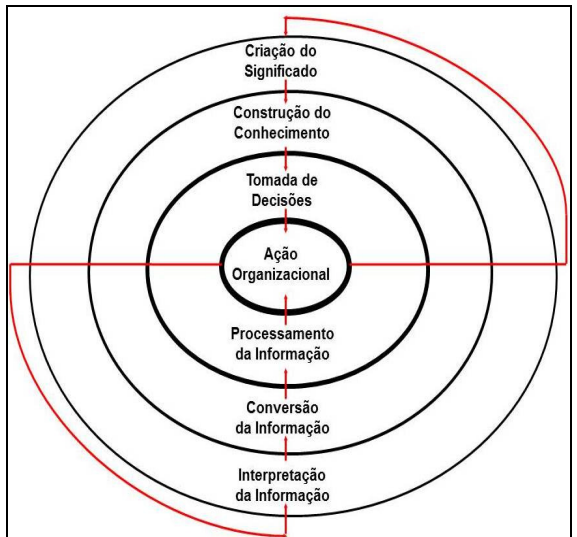

Fonte: Choo (2003, p. 31). 
Em cada arena há a presença de um contexto sociocultural, útil para balizar a percepção do nível estratégico quanto à constituição dos problemas da organização. A proposta de Choo (2003) aproxima-se da utilidade e uso da informação no contexto organizacional e de todo o processamento necessário para tornar a informação acessível aos indivíduos, geralmente inseridos em um contexto estruturado formalmente. Desse modo, interpretar, converter e processar se inserem no âmbito cultural.

Fica evidente que a construção de conhecimento depende de um ambiente em que os indivíduos sejam preparados tanto para transformar os tipos de conhecimento, como também para participar efetivamente de processos de compartilhamento. Assim, as condições socioculturais de produção e disseminação de conhecimento passam a integrar as discussões teórico-práticas presentes na literatura sobre gestão, pois representam parte considerável das dificuldades em obter comportamentos positivos e adaptados com relação a tornar o conhecimento parte dos processos e recurso para tomada de decisões. A construção e a adaptação do comportamento dos indivíduos atuantes em ambientes organizacionais são fundamentais para efetivar diversos processos dependentes tanto da internalização como da externalização do conhecimento, entre eles os processos de mudança voltados à melhoria contínua.

Assim, o conhecimento é essencial para desenvolver as competências aptas à prática da melhoria contínua (FLEURY; OLIVEIRA, 2001; GARVIN, 1993; NONAKA; TAKEUCHI, 1997).

\subsection{Aprendizagem Organizacional na Melhoria Contínua}

No contexto atual, é amplamente reconhecido que a Gestão da Qualidade Total é fundamental para a competitividade organizacional, não sendo mais fator de diferenciação, mas requisito mínimo para a manutenção de uma empresa no mercado. O termo Gestão da Qualidade Total é usado para descrever práticas de qualidade dentro das organizações (OLIVER, 2009), amparadas em três princípios: foco no cliente, melhoria contínua e envolvimento dos funcionários.

Melhoria contínua é comumente definida como sendo um processo focado na inovação incremental e contínua em toda a empresa (CAFFYN; BESSANT, 1996; 
DEMING, 1993; GRÜTTER et al., 2002; SLACK; JOHNSTON; CHAMBERS, 2009), devendo ocorrer de cima para baixo no organograma organizacional via estímulo aos funcionários em examinar e recomendar mudanças nos métodos e processos de trabalho dos quais participam (DAVENPORT, 1994). O conceito também inclui a ideia da capacidade organizacional na resolução de problemas (BESSANT et al., 2001) e na mudança em seus processos (WU; CHEN, 2006).

Sua condução depende de uma abordagem sistemática e científica, onde o processo de resolução de problemas deve ser estruturado em etapas como: identificação das causas, escolha das alternativas, planejamento, padronização da solução, implementação e acompanhamento (SHIBA; GRAHAM; WALDEN, 1993; UPTON, 1996).

Considerando o envolvimento das pessoas como fundamental para a melhoria contínua (BESSANT et al., 2001; CAFFIN; BESSANT, 1996; DAVENPORT, 1994; SHIBA; GRAHAM; WALDEN, 1993), vários autores destacam o papel da aprendizagem organizacional como fundamental, e também dinamizador, desse envolvimento (ANAND et al., 2009; BESSANT; FRANCIS, 1999; TRANFIELD et al., 2000), afirmando que a melhoria contínua é somente alcançada quando o aprendizado ocorre de modo contínuo e sistematizado na organização.

Por ser inerente à melhoria contínua, a mudança sempre provoca o desequilíbrio na organização e, consequentemente, no cotidiano das pessoas que trabalham nela (ARAUJO, 2010). De acordo com Pinto (2007), para que haja a mudança organizacional é preciso que as pessoas ligadas à empresa entendam que tais processos de mudanças realmente são necessários para a organização. Outro ponto a ser analisado é a prontidão, ou seja, é necessário comprometimento das pessoas envolvidas com tais processos.

Diante disso, estratégias como estímulo à participação e envolvimento, educação e treinamentos voltados àqueles envolvidos na mudança são fundamentais para o bom desempenho da mesma (ROBBINS, 2000; ROBBINS; JUDGE; SOBRAL, 2011). Nisso, novamente destaca-se a importância da aprendizagem organizacional.

O conceito de aprendizagem organizacional tem sido descrito como um conjunto de processos de ações de melhoria por meio do entendimento, experiência e conhecimento dos participantes desses processos, com o objetivo de manter e 
melhorar o desempenho da organização. Com início a partir dos anos 1980, seus princípios e práticas emergem e são aplicados em diversos fatores organizacionais, tais como estratégia, cultura, estrutura, inovação, resolução de problemas e participação dos funcionários (ANTONELLO, 2005).

Para Coriat e Dosi (2002), os processos pelos quais as empresas adquirem, constroem e modificam suas capacidades são denominados de aprendizado organizacional, podendo ocorrer em dois níveis: por meio da troca de conhecimento e experiências com os membros de uma organização ou pela introdução de novos membros provedores de conhecimentos que a organização não possuía previamente.

Já para Senge (1990), as organizações nas quais as pessoas expandem de forma contínua sua capacidade de criar os resultados que desejam surgindo como consequência, novos e mais elevados padrões de raciocínio em relação ao estado anterior, podem ser denominadas como organizações que aprendem. Aqui vale uma ressalva em relação ao termo "organizações que aprendem". Embora utilizado nos estudos seminais sobre 0 assunto, pela dificuldade das organizações em alcançarem esse estado, o termo foi sendo suplantado para "aprendizagem organizacional", mais utilizado nos dias atuais (ANTONELLO, 2005).

Ainda segundo Senge (1990), as organizações só aprendem por intermédio de indivíduos: o aprendizado individual não garante o organizacional, mas sem aquele não há como este ocorrer. Para Versiani (2006), a aprendizagem organizacional cria condições para que as melhorias aconteçam posto ser um processo que compreende todas as dimensões das mudanças organizacionais, desde sua formulação estratégica até o envolvimento das pessoas nos grupos de trabalho.

A literatura que aborda a temática da aprendizagem organizacional possui forte influência oriunda da melhoria contínua (OLIVER, 2009; SCARBROUGH; SWAN; PRESTON, 1998; TERZIOVSKI et al., 2000). Isso porque um dos pilares da melhoria contínua está em conhecer e satisfazer as necessidades dos clientes, forçando mudanças internas nos produtos, processos, o que por sua vez leva à necessidade de conhecimento de novos métodos, procedimentos e padrões de trabalho e comportamento. Para Wang e Ahmed (2002), a adoção da gestão da qualidade total é um marco para uma organização de aprendizagem. 
Garvin (1993) afirma haver forte ligação entre a melhoria contínua e a aprendizagem organizacional, propondo cinco fontes dessa aprendizagem: (i) resolução sistemática de problemas: privilegia a utilização de métodos científicos para diagnosticar problemas ao invés do tradicional "feeling"; (ii) experimentação: consiste na procura e experimentação de novos conhecimentos, utilizando métodos científicos; (iii) aprendizagem com a experiência passada: baseia-se na revisão sistemática das experiências passadas, considerando os sucessos e fracassos; (iv) aprendizagem com o ambiente externo: baseia-se na utilização de experiências externas, ganhando uma nova perspectiva com a análise das experiências vividas por outras organizações; (v) difusão do conhecimento: decorre da transferência do conhecimento por toda a organização, utilizando as técnicas de educação, treinamento, padronização para difusão desse conhecimento.

$\mathrm{O}$ autor afirma que o melhoramento contínuo requer um compromisso com a aprendizagem, em que uma organização não pode progredir sem antes aprender algo novo. Nesse mesmo raciocínio, Bessant et al. (2001) apresentam as etapas a serem seguidas para obtenção do conhecimento voltado à melhoria contínua: (a) entender os conceitos de melhoria contínua, articulando seus valores básicos, (b) desenvolver o "hábito" da melhoria contínua, por meio do envolvimento das pessoas e da utilização de ferramentas e técnicas adequadas, (c) criar um foco para a melhoria contínua pela sua ligação com os objetivos estratégicos da empresa, (d) aprender direta e indiretamente a criar procedimentos que sustentem a melhoria contínua, (e) alinhar a melhoria contínua por meio da criação de uma relação consistente entre os valores e procedimentos com o contexto organizacional (f) implementar ações voltadas para a resolução de problemas, (g) administrar estrategicamente a melhoria contínua promovendo seu aprimoramento, (h) desenvolver a capacidade de aprendizado de como fazer a melhoria contínua em todos os níveis e funções.

Anand et al. (2009) entendem a aprendizagem organizacional como uma das principais fontes de capacitação para a melhoria contínua. Nesse estudo, os autores propõem um modelo teórico de infraestrutura para a melhoria contínua, sendo um dos elementos a aprendizagem dos funcionários envolvidos nos programas, via iniciativas de treinamento, capacitação e envolvimento. 
A melhoria contínua é apontada para obter inovação (ANTONELLO, 2005; CAFFYN, 1999; DAVENPORT, 1994; SHIBA; GRAHAM; WALDEN, 1997), tanto que o conceito de melhoria contínua tem evoluído para o de inovação contínua, entendida como a capacidade de combinar a eficiência operacional com a flexibilidade estratégica (HYLAND; BOER, 2006).

Como na sua essência inovação é um processo no qual ideias são geradas (SCHUMPETER, 1984; ROGERS, 1995), representando o resultado de uma ampla gama de processos de aprendizagem e conhecimento, justifica-se a relação dos temas melhoria contínua, inovação e aprendizagem organizacional existente na literatura.

As possibilidades de mudanças oriundas da inovação podem estar relacionadas a outras variáveis, que não somente a produto e processo (SCHUMPETER, 1988), tais como alterações na forma de organização e gestão da organização, mudanças na organização e administração do processo produtivo, incorporação de estruturas organizacionais modificadas, e implementação de novas orientações estratégicas corporativas ou substancialmente modificadas. Dessa forma, a geração ou adoção de uma inovação requer não somente um processo técnico para se obter um resultado de êxito, mas também um conjunto de competências e habilidades, assim como rotinas organizacionais internas que proporcionem as bases competitivas em um determinado mercado ou em diferentes mercados.

Possas (1989) acrescenta a esta discussão a questão dos mecanismos de aprendizado que, segundo o autor, são tipicamente de três modalidades. A primeira é representada pelo investimento em Pesquisa e Desenvolvimento (P\&D) e constitui o mais importante meio de aprendizado: a acumulação tácita de conhecimento realimenta o processo de busca de inovações e aperfeiçoamento de produtos e processos.

Os processos informais de acumulação de conhecimento tecnológico dentro das firmas representam o segundo meio de aprendizado. Estes processos de acumulação de conhecimento não envolvem nem destinação específica de recursos e nem um formato organizacional definido. Contudo, no desenvolvimento de novos produtos e processos que já tenham sido incorporados, podem ser de extrema 
importância. Como exemplo desse meio de aprendizado, pode-se citar os processos de learning by doing e learning by using ${ }^{2}$ (FORAY; LUNDVALL, 1996).

O learning by doing consiste em uma forma de aprendizado que ocorre no processo de produção e materializa-se no desenvolvimento de habilidades, na redução dos custos com mão-de-obra e na diminuição de problemas de qualidade. Por sua vez, o learning by using ocorre com a utilização do produto pelo seu usuário final, cujos resultados podem se refletir tanto em práticas de operação e manutenção mais eficazes, quanto em informações que, se repassadas para a empresa produtora, refletem na introdução de melhorias incrementais no produto (FORAY; LUNDVALL, 1996).

O terceiro meio de aprendizado é representado pelo desenvolvimento de externalidades intra e interindustriais. Essas externalidades incluem a difusão de informação, mobilidade de mão-de-obra especializada e o crescimento de serviços especializados.

O capital humano é considerado um fator de expressiva importância nos processos de inovação destacando-se, com isso, a importância da educação formal e da capacitação em instituições externas. Embora muitas vezes as empresas sejam vistas essencialmente como usuárias, e não como criadoras do capital humano, algumas habilidades e conhecimentos mais específicos são somente adquiridos no âmbito dessas empresas, por meio de seus investimentos em learning by doing e treinamento (BELL; PAVITT, 1993).

Bell (1984) afirma que o aprendizado tecnológico numa organização depende de duas variáveis: mecanismos que representam um menor custo de fabricação do produto e alocação de recursos como mecanismo sistemático de retorno de informações, treinamentos, contratação de pessoas.

Assim, na concepção de Bell (1984), há seis diferentes tipos de informação ou conhecimento que constituem o aprendizado, a saber: (i) learning by operating: consiste em uma combinação de estímulo à mudança e aumento do entendimento do processo; (ii) learning from changing: relaciona-se ao aprendizado gerado por meio de vários tipos de mudança técnica e não ao aprendizado gerado realizando-se

\footnotetext{
${ }^{2}$ As expressões learning by doing e learning by using costumam não receber tradução quando usadas pela literatura brasileira sobre inovação tecnológica. Uma tradução aproximada seria "aprender fazendo" e "aprender usando".
} 
operações; (iii) system performance feedback: envolve a aplicação de mecanismos institucionalizados para gerar, registrar, revisar e interpretar a experiência; (iv) learning through training: consiste em treinamentos formalizados que são importantes como fontes de capacidade tecnológica; (v) learning by hiring: consiste na contratação de pessoas que personifiquem as habilidades e conhecimentos já disponíveis no ambiente das empresas, (vi) learning by searching: depende do esforço ativo da organização em empregar recursos para realizar a busca de novas tecnologias que são internas à empresa, ou seja, que estão formalizadas em departamentos ou equipes ${ }^{3}$.

Nesse contexto, observa-se que os processos de aprendizado não se restringem apenas às atividades de Pesquisa e Desenvolvimento formais das empresas, mas também, e cada vez mais, na capacidade da empresa em estabelecer mecanismos de interações e de socialização, com os quais sustente as atividades geradoras e fornecedoras de conhecimento fundamentais para a mudança e melhoria contínua.

\subsection{Influência da Cultura Organizacional}

A cultura organizacional é a forma como um grupo percebe e reage a respeito de problemas internos e externos à organização. Composta de elementos e processos como valores, crenças, rituais, mitos, estratégias, socialização, liderança, comunicação e mudança, tem a função de delimitar o grupo, de facilitar a identificação dos indivíduos que pertencem a ela, de melhorar o processo de adesão e perpetuação dos padrões necessários para a existência do grupo e de promover a orientação dos comportamentos organizacionais nos níveis individuais e grupais.

Divide-se em cultura material e cultura não-material, sendo a primeira mais fácil de identificar porque é visível e mensurável como ocorre a respeito das tecnologias de informação e comunicação, de normas explícitas sobre o uso de uniformes e horário de trabalho. Por sua vez, na cultura não-material se localizam elementos não palpáveis como os valores, crenças e mitos que são origem a

\footnotetext{
${ }^{3}$ As expressões contidas nesse parágrafo também costumam não receber tradução quando usadas pela literatura brasileira sobre inovação tecnológica.
} 
comportamentos e formas de pensar particulares de um grupo. Todavia, ressalta-se que em ambas pode-se aplicar gestão (JOHANN, 2004).

Isso significa que processos de mudança podem ser aplicados à cultura organizacional, em primeiro lugar na cultura material e, posteriormente, na cultura não-material. Rituais e procedimentos normativos como processos de socialização ou treinamento são bastante uteis para promover a mudança e facilitar o aprendizado dos novos padrões.

Os fatores apresentados nos itens anteriores relacionados à melhoria contínua implicam num fato: ocorrência de mudança. Para Araujo (2010), as organizações que desejam voltar-se para a Gestão da Qualidade Total precisam aceitar e gerenciar a mudança, modificando a forma como entendem seus processos internos e sua relação externa com o mercado.

Num ambiente em constante mudança, as organizações são influenciadas pelos agentes do ambiente em que estão inseridas e pelas mudanças no modo de pensar e agir, a velha forma de se fazer as coisas deve ser substituída, adaptada a esta nova realidade. É nesse ponto que se encontra a importância da mudança de paradigma, que inclui elementos como aprendizagem, auto-reflexão e cultura organizacional (FRANCIS; BESSANT, 2005).

Mudança organizacional é comumente definida como sendo qualquer alteração significativa de estado, de modo articulado e planejado, e operacionalizada por pessoa interno e externo à organização, com apoio e supervisão da administração superior. Visa atingir os componentes de cunho comportamental, estrutural, tecnológico (equipamentos e conhecimento) e estratégico (ARAUJO, 2010; KOTTER; SCHLESINGER, 1986; ROBBINS; JUDGE; SOBRAL, 2011; WOOD JUNIOR, 1995).

A predisposição para a mudança por parte da organização depende muito das características e dos paradigmas de sua cultura organizacional. Cultura organizacional é o conjunto de valores em vigor numa empresa, suas relações e sua hierarquia, definindo os padrões de comportamento e de atitudes que governam as ações e decisões mais importantes da administração, ou ainda, as crenças em relação ao que é importante na vida e expectativas sobre o comportamento dos membros da organização (LACOMBE; HEILBORN, 2003). 
A cultura organizacional tem impacto acentuado na capacidade de mudança das organizações. Sendo um dos recursos da administração, pode ser usada para alcançar os objetivos da mesma forma que a tecnologia, os insumos de produção, os equipamentos, os recursos financeiros e humanos. Dos valores dessa cultura, pode ser priorizado em maior ou menor grau o nível de desempenho e os critérios para a sua avaliação; a capacidade de inovação e de criação; a competitividade e o espírito de lealdade (MAXIMIANO, 2006).

Mesmo em organizações onde a cultura organizacional é avessa aos processos de mudança, ressalta-se que, muito embora essa cultura esteja enraizada muitas vezes por longos períodos, ela pode e deve ser suplantada. Lacombe e Heilborn (2003) ressaltam que os executivos devem estar conscientes disso e que os administradores com conhecimento da cultura devem saber identificar o que e como deve ser mudado e quando a mudança é necessária. Agindo dessa forma, a empresa estará constantemente habilitada a enfrentar o mercado em permanentes mudanças.

As mudanças ocorridas em função da melhoria contínua necessitam de uma série de ações e recursos de apoio. Nisso, a relação da cultura organizacional com a melhoria continua se dá em dois aspectos: primeiro, na existência de uma cultura organizacional voltada à busca constante por melhoria a partir da consciência da necessidade de mudança em função das influências do mercado e da busca pela competitividade; segundo, na transferência desse estado de consciência para ações concretas, de ordem estrutural, técnica e comportamental (JAGER et al., 2004).

Diversos são os estudos que discutem os fatores facilitadores e iniciativas de suporte para os programas de melhoria contínua (ANAND et al., 2009; JACA et al., 2012; POIRIER; HOUSER, 1993; MARIN-GARCIA; PARDO-DEL-VAL; BONAVIA, 2008).

No modelo para a gestão da melhoria contínua proposto por Poirier e Houser (1993), a "análise da cultura da organização" é a primeira entre as três fases do modelo, cujo objetivo consiste em analisar as condições atuais da organização e combinar seus propósitos e valores com as intenções do processo de melhoria. Os autores destacam a importância do envolvimento das pessoas nessa etapa.

Jaca et al. (2012) levantam os fatores num total de quinze e os chamam de "fatores de sustentabilidade para a melhoria contínua". Entre eles, os ligados com a 
existência de uma cultura organizacional favorável são: (a) comprometimento e envolvimento da gestão; (b) alinhamento dos objetivos dos programas de melhoria com os objetivos estratégicos da organização; (c) uso de recursos apropriados aos programas de melhoria, tais como financeiros, estruturais, de tempo; (d) envolvimento da força de trabalho nos programas de melhoria; (e) treinamento adequado; (f) comunicação dos resultados obtidos com os programas para o restante da organização; $(\mathrm{g})$ envolvimento de outras pessoas, além das formalmente atribuídas aos programas; (h) fornecimento de facilitadores, como por exemplo, pessoas na função de mediadoras; (i) adaptação às forças ambientais e (j) reconhecimento e recompensa aos participantes.

O trabalho de Anand et al. (2009) também reforça o papel da cultura organizacional para o desenvolvimento dos programas de melhoria contínua, no caso apresentando-a como um dos elementos da "infraestrutura para a melhoria contínua". Na visão dos autores, a cultura organizacional deve promover a cultura interna para a constante mudança por parte dos participantes da organização. Essa cultura interna torna-se então uma subcultura da cultura geral da organização, onde os funcionários devem ser preparados para mudanças e reorientações, além de terem a capacidade para a percepção da necessidade dela.

$\mathrm{Na}$ lista de fatores facilitadores dos programas de melhoria contínua apresentados num estudo empírico realizado por (MARIN-GARCIA; PARDO-DELVAL; BONAVIA. 2008), a cultura organizacional de estímulo à criatividade se destaca como importante.

Para que a cultura direcionada à mudança de fato exista numa organização, é necessário que os funcionários estejam predispostos a isso. Portanto, os aspectos psicológicos e comportamentais que envolvem receios, esperanças, desejos e frustrações relativas à adoção e utilização de tecnologias por parte dos profissionais, resultado de qualquer processo de mudança, representam um dos fatores que podem facilitar ou impedir essa mudança.

Diante disso, é imprescindível que uma organização tenha sua cultura organizacional favorável à mudança, com normas e regulamentos que a incentivem e clima organizacional apto a lidar com esses processos de natureza fundamentalmente humana/comportamental, e não apenas técnico/estruturais 
(BORGES; ALBUQUERQUE, 2004; FERGUSON; REIO JUNIOR, 2010; FRANCIS; BESSANT, 2005; KRAATZ; ZAJAC, 2001).

\section{MÉTODO DE PESQUISA}

Para atender ao objetivo proposto foi escolhida uma abordagem qualitativa, desenvolvida por meio do método de estudo de caso e seguindo o paradigma fenomenológico. Essas escolhas foram sustentadas pelo problema de pesquisa, cuja intenção foi acompanhar resultados de um novo procedimento implantado na organização. Desse modo, no paradigma fenomenológico pretende-se compreender o fenômeno a partir da perspectiva subjetiva e construída socialmente pelo grupo ou indivíduo investigado, não recorrendo à aplicação de testes de hipótese, de controle de variáveis e de análises estatísticas de correlação entre variáveis.

Temas e problemas do âmbito sociocultural geralmente se associam à pesquisa qualitativa. Desde a década de 1990 a abordagem qualitativa vem ganhando espaço na pesquisa em Administração (VIEIRA; ZOUAIN, 2006), sugerindo necessidade de efetivar análises não restritas a dados descritivos, comparando e abstraindo a partir de uma matriz teórica adotada. Nesse caso, temse como pressuposto elaborar uma compreensão particular do objeto estudado (MARTINS; BICUDO apud COLTRO, 2000). A validação da análise recorre à capacidade de interpretação lógica do pesquisador e á fundamentação teórica, tal como adotado na presente pesquisa.

A pesquisa bibliográfica foi útil na medida em que proporcionou conhecimentos prévios sobre o tema. Conceitos e compreensões gerais foram necessários, entretanto, isso não caracterizou o uso de modelos teóricos rígidos, testados junto ao fenômeno. Por outro lado, as informações coletadas foram expostas à literatura pesquisada, procedimento que confirmou a necessidade de interpretação.

O objeto de estudo foi uma empresa do ramo de serviços em telecomunicações do interior do estado de São Paulo, atuante há mais de 10 anos no mercado. Atualmente conta com 60 funcionários distribuídos entre matriz e duas filiais. Seus serviços são oferecidos para o público residencial e corporativo. 
Foram verificadas informações a respeito do processo de mudança visando a melhoria na qualidade dos serviços prestados, desenvolvido pela empresa entre os anos 2010-2011. Para a coleta de dados foi utilizado como instrumento um roteiro de entrevista elaborado na forma de questionário semi-estruturado, contendo questões abertas e fechadas, aplicado pessoalmente aos funcionários de nível operacional e gerencial envolvidos nos projetos. Isso significa que a entrevista foi a técnica aplicada e o roteiro foi o tipo de instrumento.

A fim de preservar a identidade da empresa, o projeto é descrito como "Projeto X". Algumas informações mais detalhadas do projeto, que pudessem caracterizar os procedimentos técnicos utilizados, são também omitidas. Essas omissões não prejudicam o desenvolvimento deste trabalho, posto que o objetivo é analisar como a gestão do conhecimento e a cultura organizacional favorecem os processos de mudança visando a qualidade e melhoria contínua. Nesse caso, aplicou-se a Apresentação da Pesquisa aos sujeitos, com o intuito de resguardar os direitos e sua a escolha em participar da pesquisa, procedimento este sugerido pela Resolução 196/1996 do Conselho Nacional de Saúde.

Os próximos subitens tratarão da apresentação e discussão do projeto em análise, comparando os resultados obtidos com o referencial teórico apresentado.

\section{APRESENTAÇÃO E DISCUSSÃO DOS RESULTADOS}

O projeto $\mathrm{X}$ visava à obtenção de uma certificação de qualidade específica da área de atuação da empresa, com o objetivo de agregar maior diferencial competitivo perante o mercado. Por não possuir essa certificação, a empresa estava encontrando dificuldades em conquistar novos clientes corporativos e em manter os atuais. Portanto, o principal motivador para essa mudança visando a melhoria foi o mercado.

A situação enfrentada pela empresa corrobora com o cenário atual apontado na revisão de literatura. Como as organizações têm sofrido fortes imposições do mercado, a busca pela melhoria contínua está se tornado comum como meio para o aumento da competitividade e sobrevivência sustentável (ZAMPINI; TOLEDO, 2010).

Havia um prazo de início e fim determinados pela certificadora e o seu não cumprimento, bem como o insucesso da implementação, prejudicaria a empresa na 
conquista de novos clientes, na manutenção dos clientes atuais e na imagem perante o mercado, principalmente considerando a concorrência. Além desses, haveria também prejuízos financeiros, pois tanto a certificadora quanto as mudanças internas oriundas do projeto, exigiram altos investimentos.

A primeira etapa para o início do projeto foi a comunicação de que ele seria realizado, os objetivos e benefícios pretendidos, os motivos impulsionadores, quais setores e pessoas estariam diretamente envolvidos e quais mudanças, até aquele momento possíveis de serem previstas, ocorreriam. Os primeiros as serem comunicados e conscientizados foram os gestores das quatro áreas diretamente envolvidas e, posteriormente, os demais funcionários de nível operacional dessas áreas.

Reuniões foram utilizadas na organização como instrumento de acesso às informações sobre o projeto, estimulando discussões, participação e adesão. O intuito era diminuir as restrições iniciais às mudanças ao promover claro entendimento dos objetivos do projeto e conscientizar os envolvidos da necessidade dela. A intenção da empresa era, de fato, envolver as pessoas: apesar de a mudança estar imprescindível, os gestores da alta administração não queriam que fosse conduzida e implementada de modo imposto. Como resultado, as equipes aceitaram o projeto e se predispuseram para que fosse realizado no prazo estimado e com excelência. Posteriormente, todas as demais áreas e pessoas foram também comunicadas.

As ações realizadas pela empresa para o envolvimento das pessoas no projeto desenvolvido são apontadas pela literatura como essenciais nas ações de melhoria contínua (ARAUJO, 2010; BESSANT et al., 2001; CAFFIN; BESSANT, 1996; DAVENPORT, 1994; PINTO, 2007; SHIBA; GRAHAM; WALDEN, 1993).

É válido ressaltar que esse posicionamento por parte da alta administração é oriundo de experiências anteriores, quando a falta de comunicação, envolvimento em termos de aceitação e treinamento técnico para a mudança fez com que projetos fossem mal-sucedidos financeiramente e operacionalmente. Diversos modelos e estudos sobre os facilitadores para melhoria contínua prevêem o comprometimento da alta administração como aspecto fundamental para o sucesso das iniciativas (KAYE; ANDERSON, 1999; MARIN-GARCIA; PARDO-DEL-VAL; BONAVIA, 2008; POIRIER; HOUSER, 1993). 
Com o entendimento das equipes quanto ao projeto, os processos de mudanças tiveram início. As mudanças foram de ordem tecnológica (investimento em estrutura, máquinas e equipamentos, softwares), processuais (métodos de trabalho, etapas e sequenciamento das atividades) e comportamentais (padrões de comportamento dos funcionários na realização do trabalho e na relação com a empresa e cliente).

As mudanças tecnológicas exigiram estudos a fim de entender como seriam realizadas e como não prejudicariam os demais setores durante a fase de transição. O conhecimento prévio da equipe foi importante nesse momento. As mudanças referentes aos processos de trabalho dependeram de cada membro individualmente, de seu entendimento e ciência dos novos métodos a serem adotados nas duas naturezas que compõem a relação das pessoas com o trabalho: técnica e comportamental.

Neste momento, ressalta-se a importância das organizações, nos seus projetos de melhoria, em promover a comunicação e a capacitação dos funcionários e, que para isso, é preciso ter cultura organizacional voltada à melhoria contínua (GARVIN, 1993; JAGER et al., 2004).

A princípio a equipe realizou um relatório apresentando quais processos eram utilizados até então. A partir deste, identificou-se os processos em duplicidade e desnecessários. Dos que restaram, analisaram-se quais deles poderiam ser unificados, para diminuir as etapas de trabalho, e adaptados às novas políticas da empresa. Estas mudanças foram de impacto significativo, posto que ocasionaram alterações de procedimentos realizados diariamente desde o início da empresa. Portanto, houve mudanças também nas normas e política da empresa.

Percebe-se que os membros da equipe foram estimulados a fazer uso de seus conhecimentos, em especial o conhecimento explícito (NONAKA; TAKEUCHI, 2008). Os novos procedimentos estabelecidos resultaram em um novo “conhecimento organizacional” (PÉREZ-MONTORO GUTIÉRREZ, 2008).

Apesar de todo empenho em comunicar e envolver as equipes houve resistência por parte de alguns funcionários para os novos padrões comportamentais exigidos, pois se tratava de hábitos permitidos há certo tempo e antes considerados corretos. No entanto, essa é uma situação natural e inerente a qualquer processo de mudança. A literatura aponta que a gestão humana nesses processos e a existência 
de cultura organizacional sólida para mudança, voltada à melhoria, minimizam essas reações e efeitos, mas não as impedem. Em todo processo de mudança, sempre haverá forças restritivas e impeditivas (SCHEIN, 1989).

No entanto, essas resistências não foram impeditivas. O projeto chegou ao seu término antes do prazo máximo estipulado e os resultados oriundos da certificação puderam ser percebidos no curto prazo. A empresa também sentiu efeitos positivos indiretos: mesmo as áreas não diretamente envolvidas melhoram seu desempenho. Isso se justifica pelo caráter sistêmico de toda organização, em que mudanças ocorridas em qualquer área afetam as demais (BERTALANFFY, 1976).

Houve alto envolvimento das equipes nos desafios e problemas ao longo do projeto, sendo que o conhecimento prévio dos integrantes foi essencial na solução dos mesmos. Apesar dos conhecimentos técnicos prévios dos funcionários, a empresa também possibilitou novos conhecimentos via treinamento e encontros de discussão. Como a mudança resultou em novos procedimentos de trabalho, inclusive pelo uso de novas tecnologias, a capacitação foi necessária.

Com isso, a empresa utilizou-se de três formas de aprendizado apontadas por Bell (1984): learning by operating (combinação de estímulo à mudança e aumento do entendimento do processo), learning from changing (relaciona-se ao aprendizado gerado por meio de vários tipos de mudança técnica) e learning through training (consiste em treinamentos formalizados que são importantes como fontes de capacidade tecnológica).

Por parte dos envolvidos, essa capacitação foi entendida como apoio da alta administração às suas limitações diante do desconhecido, o que ajudou a diminuir as resistências e a aumentar o envolvimento não apenas com o projeto, mas com a empresa como um todo.

Outra informação possível de ser observada no caso é a presença da cultura interna favorável à mudança, busca pela maior qualidade e inovação, bem como estímulo ao aprendizado frequente da força de trabalho da empresa via treinamentos e outras estratégias de capacitação. Isso se dá pelo fato de pertencer a um ramo (telecomunicações) em constantes transformações e mutações. Desde sua fundação, a empresa tem sido pioneira em projetos de oferta de novos produtos e serviços, alguns deles inclusive de extensão nacional. 
Andréia de Abreu; Cássia Regina Bassan de Moraes; Luana Maia Woida Gestão do conhecimento como apoio à melhoria contínua: um estudo de caso em uma empresa de telecomunicações

O Quadro 1 sintetiza as informações obtidas na análise do caso:

Quadro 1 - Informações sobre o projeto de melhoria para obtenção de uma certificação de qualidade específica da área de atuação da empresa

\begin{tabular}{|c|c|}
\hline Instrumentos utilizados & $\begin{array}{l}\text { - } \text { reuniões de comunicação do projeto } \\
\text { - } \quad \text { reuniões de acompanhamentos } \\
\text { - } \text { divisão de tarefas e atribuição de responsabilidades } \\
\text { - } \quad \text { treinamento e capacitação dos funcionários envolvidos } \\
\text { - estímulo ao trabalho em equipe }\end{array}$ \\
\hline $\begin{array}{c}\text { Natureza das mudanças } \\
\text { internas }\end{array}$ & $\begin{array}{l}\text { - } \text { tecnológica, processual e comportamental } \\
\text { - mudança na política e normas da empresa } \\
\text { - introdução de novos métodos de trabalho } \\
\text { - eliminação de processos desnecessários }\end{array}$ \\
\hline Dificuldades & $\begin{array}{l}\text { - resistência por parte de alguns funcionários em função } \\
\text { dos desafios e problemas ao longo do projeto } \\
\text { - resistência por parte de alguns funcionários em função } \\
\text { dos novos métodos de trabalho oriundos da } \\
\text { implementação da certificação }\end{array}$ \\
\hline Benefícios & $\begin{array}{l}\text { - melhoria da imagem perante o mercado } \\
\text { - conquista de novos clientes corporativos } \\
\text { - maior facilidade de manutenção dos clientes atuais pelo } \\
\text { aumento na confiabilidade de qualidade que a } \\
\text { certificação proporciona } \\
\text { - repercussão positiva no desempenho operacional de } \\
\text { outras áreas não envolvidas no projeto } \\
\text { - melhoria no desempenho operacional } \\
\text { - aumento da participação e envolvimento dos funcionários } \\
\text { - } \text { copós o projeto } \\
\text { compartilhamento de conhecimento }\end{array}$ \\
\hline
\end{tabular}

Fonte: Elaborado pelas autoras.

Os resultados sugerem que o envolvimento das pessoas nas mudanças ocasionadas pelo projeto, via valorização do seu conhecimento e estímulo ao aprendizado durante o processo, tiveram influência significativa para o sucesso da implementação e, também, na minimização das resistências oriundas. Para Santiago Junior (2004), o entendimento sobre a importância do conhecimento em uma organização passa, necessariamente, pela clareza de alguns conceitos, dentre os quais o de criação e registro dos conhecimentos relevantes para organização e o de mapeamento das pessoas que possuem esses conhecimentos. 


\section{CONSIDERAÇÕES FINAIS}

A consciência da importância em buscar a melhoria contínua tem se tornado comum por parte das empresas. Apesar de se constituir num conceito simples, de fácil entendimento e de baixo nível de investimento, a melhoria contínua muitas vezes não tem sido implementada com sucesso (BESSANT et al., 1994).

Entre os fatores de insucesso estão a incapacidade das empresas em gerenciar processos de mudança e também a ausência de uma cultura voltada ao aprendizado e ao compartilhamento constante, tal como exige a gestão do conhecimento. Conforme aponta Bessant et al. (2001) e Juran (1995), melhoria contínua é um processo gradual de aprendizagem organizacional, onde ruptura e controle são elementos essenciais.

Portanto, é fundamental que a cultura e a infra-estrutura da organização apoiem a melhoria contínua bem como haja uso de métodos e ferramentas que facilitem a sua implementação (BESSANT et al.,1994). Consequentemente, é preciso considerar e gerenciar as competências profissionais existentes na organização a fim de garantir que sejam vistas em sua totalidade e utilizadas na prática da melhoria contínua (ANAND et al., 2009; OLIVER, 2009).

Este trabalho propôs analisar como a gestão do conhecimento contribuiu para o envolvimento das pessoas durante um processo de mudança organizacional voltado à melhoria contínua em uma empresa do ramo de telecomunicações.

As situações encontradas na pesquisa de campo corroboram com o apontado na literatura sobre o tema. As mudanças nem sempre são bem vistas por todos os funcionários de uma organização, pois as mesmas causam reações como insegurança, receio e medo pela mudança de status quo. Por sua vez, esses fatores podem gerar resistências impeditivas à mudança proposta e a gestão do conhecimento pode ser usada como ferramenta contra essas resistências: o entendimento e conhecimento sobre os motivos da mudança minimizam as resistências e aumentam o envolvimento das pessoas.

A gestão do conhecimento forja maior envolvimento dos indivíduos nos processos de construção de conhecimento, incentiva comprometimento com ações de externalização, cujos comportamentos provêm de uma base sociocultural favorável à adaptação e à mudança. 
Andréia de Abreu; Cássia Regina Bassan de Moraes; Luana Maia Woida

Gestão do conhecimento como apoio à melhoria contínua: um estudo de caso em uma empresa de telecomunicações

Para que as mudanças na empresa ocorressem foi necessária a quebra de paradigmas, suplantando alguns padrões existentes desde sua fundação. Nota-se que no desenvolvimento do projeto houve intensa interação entre seus funcionários e aceitação das mudanças por meio da comunicação e esforço da alta administração em promover o entendimento da necessidade da mudança e a melhoria resultante dela. Outro aspecto importante foi o reconhecimento do conhecimento prévio dos membros das equipes, estimulando-os nas resoluções dos problemas e na geração de ideias, e também a capacitação para as novas atividades oriundas da mudança.

Destaca-se, igualmente, o envolvimento e comprometimento da alta administração durante o projeto. Conforme apontam Dooley e O'Sullivan (1999), uma das maiores causas de insucesso das iniciativas de programas de qualidade é, justamente, a falta de comprometimento da alta administração, o que não ocorreu na empresa em estudo. Todos esses fatores contribuíram para que essa quebra de paradigmas ocorresse mais facilmente e o projeto fosse implementado com sucesso.

A prática de melhoria contínua encontrada na organização se assenta sobre os padrões da gestão do conhecimento, como representa a Figura 2. 
Figura 2 - Prática da melhoria contínua viabilizada pela gestão do

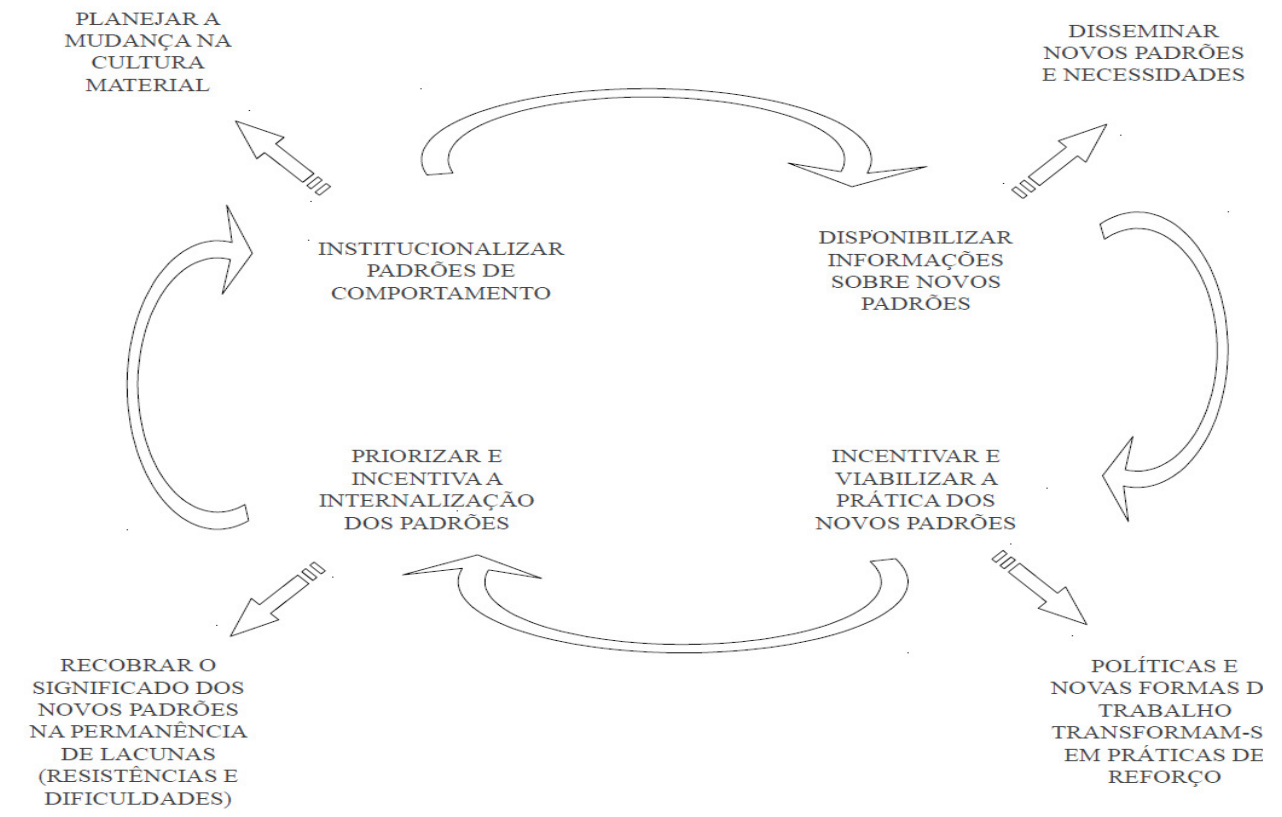

Fonte: Elaborado pelas autoras

No primeiro estágio das práticas de melhoria contínua viabilizadas pela gestão do conhecimento definem-se padrões de comportamento, provocando mudanças na cultura organizacional (material). Nos procedimentos internos adotados pela organização pesquisada, encontram-se ações específicas que indicam a institucionalização de padrões planejados, uma vez que constam modificações significativas no aumento de participação e no envolvimento dos funcionários.

Posteriormente à institucionalização ou definição, encontra-se a disponibilização de informações cuja função é disseminar e comunicar os novos padrões considerados corretos, estimulando processos de aprendizagem e de construção de conhecimento. Reuniões foram utilizadas com essa finalidade, favorecendo o processo de identificação de informações e criação de significados para a construção de conhecimento e de novos comportamentos.

Como terceiro estágio, as políticas antes planejadas submergem na organização por meio de treinamentos, práticas e rituais de reforço dos novos padrões, incidindo sobre políticas, divisão de tarefas e de responsabilidades. Os reflexos da aderência aos novos padrões manifestaram-se nos comportamentos dos funcionários, caso contrário se tornaria visível a não aceitação e as lacunas 
Andréia de Abreu; Cássia Regina Bassan de Moraes; Luana Maia Woida

Gestão do conhecimento como apoio à melhoria contínua: um estudo de caso em uma empresa de telecomunicações

deixadas pelo segundo estágio, realizado para conscientizar os envolvidos. Neste ultimo estágio, verificam-se a aderência ou não aos novos padrões, priorizando e incentivando a internalização, reduzindo resistências e dificuldades geralmente vinculadas aos aspectos socioculturais, ou perpetuando comportamentos apropriados.

Por meio de um novo planejamento dos comportamentos adequados para a prática de melhoria contínua, percebeu-se existir verificação constante da necessidade de mudanças na cultura e no comportamento, pois o intuito é incentivar maior participação e envolvimento das pessoas na prática de melhoria contínua, baseando-se no comprometimento, na externalização e construção de conhecimento.

Acredita-se que esse estudo proporciona melhor compreensão da gestão do conhecimento e envolvimento das pessoas na melhoria contínua. As informações obtidas no estudo de caso permitiram conhecer como um projeto bem sucedido de mudança para a melhoria foi conduzido, permitindo a comparação da teoria com a prática. Portanto, além da contribuição teórico-acadêmica com a revisão de literatura, há também a contribuição empírica, que pode servir como exemplo para os gestores que venham a atuar em iniciativas de melhoria contínua.

\section{REFERÊNCIAS}

ANAND, Gopesh et al. Dynamic capabilities through continuous improvement infrastructure. Journal of Operations Management, Amsterdam, v. 27, p. 444-461, 2009.

ARAUJO, Luiz Cesar G. Organização, sistemas e métodos e nas tecnologias de gestão organizacional. 3. ed. São Paulo: Atlas, 2010. 1 v.

ANTONELLO, Claudia Simone. A metamorfose da aprendizagem organizacional: uma revisão crítica. In: RUAS, Roberto; ANTONELLO, Claudia Simone; BOFF, Luiz Henrique (Org.). Os novos horizontes da gestão: aprendizagem organizacional e competências. Porto Alegre: Artmed, 2005.

BELL, Martin; PAVITT, Keith. The development of tecnological capabilities. In: BELL, Martin et al. Trade, technology and international competitiveness. Waschington: World Bank, 1995. p. 69-102. 
BELL, Martin. Learning and the accumulation of industrial technological capacity in development countries. In: FRANSMAN, Martin; KING, Kenneth (Ed.).

Technological capability in the third world. London: Macmillan Press, 1984. p. 187-209.

BERTALANFFY, Ludwig von. Teoria dos sistemas. Rio de Janeiro: FGV, 1976.

BESSANT, John; CAFFYN, Sarah; GALLAGHER, Maeve. An evolucionary model of continous improvement behaviour. Technovation, Ottawa, v. 21, n. 1, p. 67-77, 2001.

BESSANT, John; FRANCIS, David. Developing strategic continuous improvement capability. International Journal of Operations \& Production Management, United Kingdom, v. 19, n. 11, p. 1106-1119, 1999.

BESSANT, John et al. Rediscovering continuous improvement. Technovation, Ottawa, v. 14, n. 1, p. 17-29, 1994.

BORGES, Livia de Oliveira; ALBUQUERQUE, Francisco J. B. Socialização organizacional. In: ZANELLI, Jose Carlos; BORGES-ANDRADE, Jairo Eduardo; BASTOS, Antonio Virgílio Bittencourt (Org.). Psicologia, organizações e trabalho no Brasil. Porto Alegre: Artmed, 2004.

CAFFYN, Sarah. Development of a continuous improvement selfassessment tool. International Journal of Operations \& Production Management, United Kingdom, v.19, n.1, p.1138-1153, 1999.

CAFFYN, Sarah; BESSANT, John. A capability-based model for continuous improvement. In: INTERNATIONAL CONFERENCE OF THE EUROMA, $3^{\text {th }}$, London. Proceedings... London, 1996.

$\mathrm{CHOO}$, Chun Wei. A organização do conhecimento: como as organizações usam a informação para criar significado, construir conhecimento e tomar decisões. São Paulo: SENAC, 2003.

COLTRO, Alex. A fenomenologia: um enfoque metodológico para além da modernidade. Caderno de Pesquisas em Administração, São Paulo, v. 1, n. 11, jan./mar. 2000.

CORIAT, Benjamin; DOSI, Giovanni. The nature and accumulation of organizational competences/capabilities. Revista Brasileira de Inovação, Campinas, v. 1, n. 2, p. 275-326, 2002.

DAVENPORT, Thomas H.; PRUSAK, Laurence. Conhecimento empresarial: como as organizações gerenciam seu capital intelectual. Rio de Janeiro: Campus, 1998.

DAVENPORT, Thomas H. Reengenharia de processos. Rio de Janeiro: Campus, 1994.

DEMING, William Edwards. The new economics: for industry, government, education. Cambridge: MIT Center for Advanced Engineering Study, 1993. 
DOOLEY, Lawrence; O'SULLIVAN, David. Decision support system for the management of systems change. Technovation, Ottawa, v. 19, n. 8, p. 483-493, 1999.

FERGUSON, Karen L.; REIO JUNIOR, Thomas G. Human resource management systems and firm performance. Journal of Management Development, Bradford, v. 29, n. 5, p. 471-494, 2010.

FLEURY, Maria Tereza Lemes; OLIVEIRA JUNIOR, Moacir de Miranda. Gestão estratégica do conhecimento: integrando aprendizagem, conhecimento e competências. São Paulo: Atlas, 2001.

FORAY, Dominique; LUNDVALL, Bengt-Ake. The knowledge-based economy: from the economics of knowledge to the learning economy. In: ORGANISATION FOR ECONOMIC CO-OPERATION AND DEVELOPMENT - OCDE (Ed.).

Employment and growth in the knowledge economy. Paris: OCDE, 1996. p. 115-121.

FRANCIS, Davi; BESSANT, John. Targeting innovation and implications for capability development. Technovation, Ottawa, v. 25, p.171-183, 2005.

GARVIN, David. Building a learning organization. Harvard Business Review, Boston, v. 71, n. 4, p. 78-91, 1993.

GRÜTTER, Anton W.; FIELD, Joy M.; FAULL, Norman H. B. Work team performance over time: three case studies of South African manufacturers. Journal of Operations Management, Amsterdam, v.20, n.5, p.641-657, 2002.

HYLAND, Paul; BOER, Harry. A continuous innovation framework: some thoughts for consideration. In: CINET CONFERENCE, $7^{\text {th }}, 2006$, Lucca. Proceedings... Lucca, 2006.

JACA, Carmen et al. Components of sustainable improvement systems: theory and practice. The TQM Journal, United Kingdom, v. 24, n. 2, p. 142-154, 2012.

JAGER, Burtus de et al. Enabling continuous improvement: a case study of implementation. Journal of Manufacturing Technology Management, Michigan, $v$. 15, n. 4, p. 315-324, 2004.

JOHANN, Silvio Luiz. Gestão da cultura corporativa: como as organizações de alto desempenho gerenciam sua cultura corporativa. São Paulo: Saraiva, 2004.

JURAN, Juran M. Mangerial breakthrough. New York: McGrawHill, 1995.

KAYE, Mike; ANDERSON, Rosalyn. Continuous improvement: the ten essential criteria. International Journal of Quality \& Reliability Management, Australia, v.16, n.5, p.485-506, 1999.

KRAATZ, Matthew S.; ZAJAC, Edward J. How organizational resources affect strategic change and performance in turbulent environments: theory and evidence.

Organization Science, New York, v. 12, n. 5, p. 632-657, 2001. 
KOTTER, John; SCHLESINGER, Leonard A.A escolha de estratégia para

mudanças. São Paulo: Nova Cultura, 1986. (Coleção Harvard de Administração, v. 7).

LACOMBE, Franscisco; HEILBORN, Gilberto. Liderança e cultura organizacional. In: Administração: princípios e tendências. São Paulo: Saraiva, 2003. Cap.

18, p. 346-365.

MARIN-GARCIA, Juan. A.; PARDO-DEL-VAL, Manuela.; BONAVIA, Tomas. Análisis de programas de mejora continua: Un estudio longitudinal en una empresa industrial. Revista Gestão \& Produção, São Carlos, v. 15, n. 3, p. 433-447, 2008.

MAXIMIANO, Antonio Cesar Amaral. Significado da administração. In:

Teoria geral da administração: da revolução urbana à revolução digital. 6. ed. São Paulo: Atlas, 2006. p. 3-23.

NONAKA, Ikujiru; TAKEUCHI, Hirotaka. Teoria da criação de conhecimento na empresa. In: Gestão do conhecimento. Porto Alegre: Bookman, 2008.

NONAKA, Ikujiru; TAKEUCHI, Hirotaka Criação de conhecimento na empresa. Rio de Janeiro: Campus, 1997.

OLIVER, Judy. Continuous improvement: role of organisational learning mechanisms. International Journal of Quality \& Reliability Management, Australia, v. 26, n. 6, p. 546-563, 2009.

PAY, Rick. Everybody's jumping on the lean bandwagon, but many are being taken for a ride. Industry Week, Cleveland, march, 2008.

PÉREZ-MONTORO GUTIÉRREZ, Mario. Gestión del conocimiento en las organizaciones. Gijón: Ediciones Trea, 2008.

PINTO, Eder Paschoal. Gestão empresarial: casos e conceitos de evolução organizacional. São Paulo: Saraiva, 2007.

POIRIER, Charles. C.; HOUSER, William. F. Business partnering for continuous improvement. San Francisco: Berret-Koehler Publishers, 1993.

POSSAS, Mário Luiz. Em direção a um paradigma microdinâmico: a abordagem neo-schumpteriana. In: AMADEO, E. (Org.). Ensaios sobre economia política moderna: teoria e história do pensamento econômico. São Paulo: Marco Zero, 1989.

ROBBINS, Stephen Paul. Administração: mudanças e perspectivas. São Paulo: Saraiva, 2000.

ROBBINS, Stephen Paul; JUDGE, Timothy A.; SOBRAL, Filipe. Fundamentos do comportamento organizacional. 14. ed. São Paulo: Pearson, 2011.

ROGERS, Everett M. Diffusion of innovations. 5. ed. New York: The Free Press, 1995. 
Andréia de Abreu; Cássia Regina Bassan de Moraes; Luana Maia Woida Gestão do conhecimento como apoio à melhoria contínua: um estudo de caso em uma empresa de telecomunicações

SANTIAGO JUNIOR, José Renato Sátiro. Gestão do conhecimento: a chave para o sucesso empresarial. São Paulo: Novatec, 2004.

SCARBROUGH, Harry; SWAN, Jacky; PRESTON, J. Knowledge management: a literature review. London: Institute of Personnel and Development, 1998.

SCHEIN, Edgar H. Organizational culture and leadership: a dynamic view. São Francisco: Jossey Bass, 1989.

SCHUMPETER, Joseph Alois. A teoria do desenvolvimento econômico. 3. ed. São Paulo: Nova Cultural, 1988.

SCHUMPETER, Joseph Alois. O processo de destruição criadora. In:

Capitalismo, socialismo e democracia. Rio de Janeiro: Zahar, 1984.

SENGE, Peter. A quinta disciplina: arte e prática da organização de aprendizagem. São Paulo: Best Seller, 1990.

SHIBA, Shoji; GRAHAM, Alan; WALDEN, David. TQM: quatro revoluções na gestão da qualidade. Porto Alegre: Artes Médicas, 1997.

A new american TQM. Productivity, 1993.

SILVA, Sergio L.; ROZENFELD, Henrique. Proposição de um modelo para avaliar a gestão do conhecimento no processo de desenvolvimento de produtos. Ciência da Informação, Brasília, v. 36, n. 1, p. 147-157, 2007.

SLACK, Nigel; JOHNSTON, Robert; CHAMBERS, Stuart. Administração da Produção. 3. ed. São Paulo: Atlas, 2009.

TERRA, José Claudio Cyrineu ; GORDON, Cindy. Portais Corporativos: a revolução na Gestão do Conhecimento. São Paulo: Negócio Editora, 2011.

TERRA, José Claudio Cyrineu. Gestão do conhecimento: o grande desafio empresarial. 2 ed. São Paulo: Negócio Editora, 2001.

TERZIOVSKI, Milè et al. Establishing mutual dependence between TQM and the learning organization: a multiple case study analysis. The Learning Organization, Inglaterra, v. 7, n. 1, p. 23-31, 2000.

TRANFIELD, David et al. Organizational learning: it's just routine. Management Decision, Inglaterra, v. 38 n. 4, p. 253-260, 2000.

UPTON, David. Mechanisms for building and sustaining operations improvement. European Management Journal, London, v. 14, n. 3, p. 215-228, 1996.

VALENTIM, Marta Lígia Pomim. Informação e conhecimento em organizações complexas. In: Gestão da informação e do conhecimento no âmbito da ciência da informação. São Paulo: Polis/Cultura Acadêmica, 2008. 
Andréia de Abreu; Cássia Regina Bassan de Moraes; Luana Maia Woida

Gestão do conhecimento como apoio à melhoria contínua: um estudo de caso em uma empresa de telecomunicações

VERSIANI, Angela França. Aprendizado organizacional na internacionalização de empresas: casos na indústria calçadista brasileira. 2006. 306f. Tese (Doutorado)-Universidade de São Paulo, São Paulo, 2006.

VIEIRA, Marcelo. Milano Falcão; ZOUAIN, Deborah Moraes. Pesquisa qualitativa em administração. 2. ed. Rio de Janeiro: FVG, 2006.

WANG, Catherine L; AHMED, Pervaiz K. Learning through quality and innovation. Managerial Auditing Journal, Bradford, v. 17, n. 7, p. 417-423, 2002.

WOOD JUNIOR, Thomaz. Mudança Organizacional. São Paulo: Atlas, 1995.

WU, Chih Wei; CHEN, Chyong Ling. An integrated structuralmodel toward successful continuous improvement activity. Technovation, Essex, v.26, n. 5, p. 697-707, 2006.

ZAMPINI, Carla Simão; TOLEDO, José Carlos. Proposta para estruturação da gestão da melhoria contínua em uma fabricante de bebidas. GEPROS - Gestão da Produção, Operações e Sistemas, Bauru, n. 2, p. 107-138, abr./jun. 2010.

\section{Titulo}

Gestión del conocimiento como apoyo a la mejora continua: un estudio de caso en una empresa de telecomunicaciones

\section{Resumen}

Introducción: la gestión de mejora continua y se ha convertido común, pero las organizaciones a menudo encuentran dificultades; en la implantación de sus conceptos.

Objetivo: el objetivo de este trabajo es analizar como la gestión del conocimiento ha contribuido para el envolvimiento de las personas durante un proceso de mudanza organizacional direccionado a la mejora continua en una empresa del ramo de telecomunicaciones.

Metodología: la investigación se caracteriza dentro de un abordaje cualitativa, haciendo el uso del caso de estudio en una empresa del ramo de Servicios en telecomunicaciones, localizada en el interior del Estado de San Pablo. Para la recopilación de datos fue utilizado un rutero de entrevista en forma de cuestionario semiestructurado, aplicado personalmente a los empleados de nivel operacional y gerencial envolvidos en los proyectos.

Resultados: el análisis de los resultados indicó que el envolvimiento de las personas en mudanzas ocasionadas por el proyecto de mejora veía la valorización de su conocimiento y estímulo al aprendizado durante el proceso, tuvieron influencias significativas para el suceso de la implementación y también en la minimización de las resistencias oriundas.

Conclusiones: se concluyó que la gestión del conocimiento proporciona una mayor participación de las personas en el proceso de construcción del conocimiento, fomenta el compromiso con las acciones de externalización, cuyos comportamientos provienen de una base sociocultural favorable a la adaptación y cambio. 
Andréia de Abreu; Cássia Regina Bassan de Moraes; Luana Maia Woida

Gestão do conhecimento como apoio à melhoria contínua: um estudo de caso em uma empresa de telecomunicações

Palabras claves: Gestión de conocimiento. Mejora continua. Comprometimiento. Mudanza organizacional.

\section{Title}

Knowledge management as support to the continuous improvement: a case study in a company of telecommunications

\section{Abstract}

Introduction: the continuous improvement management has become common; however, the organizations often meet difficulties to implement the concept.

Objective: the objective of this work is to analyze how the knowledge management contributed for the involvement of people during the organizational change process toward the continuous improvement in telecommunications company.

Methodology: the survey is characterized by a qualitative approach, using the case study in a telecommunications services company located in the interior of the State of São Paulo. In order to collect data, an interview script in the form of a semi-structured questionnaire was used, individually applied to the operations and management level employees involved in the projects.

Results: the analysis of the results indicated that the involvement of people in the changes caused by the improvement project, by enhancing their knowledge and stimulating learning during the process, had a significant influence for a successful implementation and, also, in the minimization of the related opposition.

Conclusions: it can be concluded that the knowledge management provides increased involvement of the individuals in the knowledge construction processes, stimulates the commitment with externalization actions, whose behavior is from a social-cultural basis favorable to adaptation and changes.

Key-words: Knowledge management. Continuous improvement. Commitment. Organizational change.

Recebido em: 03.04.2013

Aceito em: 31.01.2014 